\title{
Impacto de una intervención sanitaria en los cuidados oculares en pacientes de la unidad de cuidados intensivos pediátricos
}

\author{
Impact of a health intervention on eye care in pediatric \\ intensive care unit patients
}

\author{
Olivia Cardozo ${ }^{1}$, Mónica Paredes$^{1}$, Yanina Zaracho ${ }^{1}$, Laura Godoy ${ }^{1}$
}

\section{RESUMEN}

Introducción: El cuidado de los ojos forma parte de los cuidados para los pacientes en las unidades de cuidados intensivos (UCI). Objetivo: analizar el impacto de una intervención sanitaria en el cumplimiento del organigrama de cuidados oculares y la incidencia de lesiones oculares en pacientes de la UCI pediátricos antes y después de una capacitación del personal sanitario. Materiales y Métodos: El estudio fue realizado en las UCI pediátricas, del Hospital general Pediátrico Niños de Acosta Nu. Se diseño un estudio cuasi experimental de antes y después. En la cohorte pre intervención (octubre-diciembre 2017) y en la cohorte post intervención (julio-diciembre 2018), se determinó la incidencia de lesiones oculares y el porcentaje de cumplimiento del organigrama, se realizó la capacitación del personal entre ambas cohortes, finalmente fueron comparados los resultados obtenidos. Se incluyeron niños de 0 a 18 años internados en las UCI pediátricos. Los datos fueron analizados en el sistema SPSSv21. Resultados: Durante el periodo de estudio ingresaron 70 pacientes en la cohorte pre - intervención y 120 después de la misma. Fueron del sexo masculino 51(58,6\%) y 63 $(52,5 \%)$, presentaron apertura ocular anormal $18(25,7 \%)$ y $19(15,8 \%)$, lesión corneal $6(8,6 \%)$ y $3(3,3 \%)$, las lesiones oculares observadas fueron erosión de la córnea en el 8,5\% (6/70) y 3,3\% (3/120), el cumplimiento del organigrama se dio en $54(77,1 \%)$ y en $120(100 \%)$ respectivamente en las cohortes pre y post intervención. Conclusión: La capacitación del personal sanitario en cuidados oculares disminuyó la frecuencia de lesiones corneales.

Palabras claves: Cuidados críticos, pediatría, cornea, profilaxis.

\begin{abstract}
Introduction: Eye care is part of patient care in intensive care units (ICU). Objective: to analyze the impact of a health intervention on compliance with eye care measures and the incidence of ocular injuries in pediatric ICU patients before and after training for healthcare personnel. Materials and Methods: The study was carried out in the pediatric ICUs of the Acosta Ñu Pediatric General Hospital. A quasi-experimental before and after study was designed. In the pre-intervention cohort (OctoberDecember 2017) and in the post-intervention cohort (JulyDecember 2018), the incidence of eye injuries and the percentage of compliance with the eye care measures were determined, staff training was carried out between both cohorts, finally the results obtained were compared. Children aged 0 to 18 years admitted to pediatric ICUs were included. The data were analyzed in the SPSSv21 system. Results: During the study period, 70 patients were admitted to the pre-intervention cohort and 120 in the post-intervention cohort. $51(58.6 \%)$ and $63(52.5 \%)$ were male, respectively, $18(25.7 \%)$ and $19(15.8 \%)$ presented abnormal eye opening, $6(8.6 \%)$ and $3(3.3 \%)$ had corneal injuries, the most common ocular lesions observed were corneal erosion in $8.5 \%(6 / 70)$ and $3.3 \%(3 / 120)$, compliance with eye care measures occurred in $54(77,1 \%)$ and $120(100 \%)$ respectively in the pre and post intervention cohorts. Conclusion: The training of healthcare personnel in eye care decreased the frequency of corneal injuries.
\end{abstract}

Keywords: Critical care, pediatrics, cornea, prophylaxis.

\footnotetext{
${ }^{1}$ Hospital General Pediátrico "Niños de Acosta Nu". San Lorenzo, Paraguay.

Correspondencia: Olivia Cardozo Correo: occs77@hotmail.com

Recibido: 28/08/2020 Aceptado:25/09/2020

Conflicto de interés: Los autores declaran no poseer conflicto de interés.

Doi: https://doi.org/10.31698/ped.47032020005
} 


\section{INTRODUCCIÓN}

La superficie externa del ojo es la parte más crucial del cuerpo expuesto al mundo exterior. El efecto protector de los tejidos perioculares, son indispensables para la protección del globo ocular. Las cejas y las pestañas, el reflejo del parpadeo, la película lagrimal son parte del mecanismo de defensa ocular del medio ambiente circundante ${ }^{(1)}$. El cuidado de los ojos forma parte de los cuidados a ser administrados a todos los pacientes en las unidades de cuidados intensivos cuyos mecanismos naturales de protección se encuentren comprometidos o dañados ${ }^{(2)}$. Entre las alteraciones oculares en pacientes ingresados en la unidad de terapia intensiva se encuentran las lesiones de la córnea. Estas pueden se superficiales como la queratitis puntata superficial, traumáticas como la abrasión corneal e infecciosas (queratitis infecciosa ulcerativa $)^{(3)}$. Estudios publicados han revelado que con mínimas intervenciones se podrían evitar las lesiones oculares en la mayoría de los pacientes en las unidades de cuidados intensivos ${ }^{(2-5)}$.

Las medidas utilizadas para el mantenimiento de la superficie ocular van desde una limpieza sencilla del párpado con apósitos estériles, hasta la implementación de protecciones de polietileno (cámara de humedad) o la administración de lubricantes en colirio o gel, además de asegurar un cierre continuo del parpado para evitar lesiones corneales por exposición ${ }^{(2,6)}$.

El hospital donde se realizó el estudio, cuenta con un organigrama de cuidados oculares para los pacientes en la terapia intensiva pediátrica. El objetivo del presente estudio fue analizar el impacto de una intervención sanitaria en el cumplimiento del organigrama y la incidencia de lesiones oculares en pacientes de la unidad de cuidados intensivos pediátricos antes y después de la implementación de una capacitación del personal sanitario de dichas unidades.

\section{MATERIALES Y MÉTODOS}

\section{Diseño y lugar de la investigación}

El estudio fue realizado en las unidades de terapia intensiva pediátrica y unidad de terapia intensiva pediátrica cardiovascular, del Hospital general Pediátrico Niños de Acosta $\tilde{N} u$, hospital universitario de tercer nivel y de referencia en Pediatría. Se diseño un estudio cuasi experimental de antes y después.

En la cohorte pre intervención (de octubre a diciembre de 2017) se determinó la incidencia de lesiones oculares y el porcentaje de cumplimiento del organigrama de cuidados oculares establecido por el servicio de oftalmología pediátrica del hospital.

Seguidamente, se realizó durante el mes de marzo del 2018 la capacitación del personal sanitario de ambas terapias en los cuidados oculares de los pacientes de acuerdo al organigrama establecido.

Posterior al periodo de capacitación, en la cohorte post intervención (julio a diciembre del 2018), se determinó la incidencia de lesiones oculares y cumplimiento del organigrama y fueron comparados con los obtenidos en la cohorte pre intervención.

\section{Descripción de la capacitación}

La capacitación consistió en clases teóricas acerca de las lesiones oculares que puede ocurrir en las terapias, debido a ciertos factores de riesgo, los métodos de cuidado ocular, así como se estudió en detalle el organigrama y como debía aplicarse para cada situación indicada en el mismo. Las clases prácticas consistieron en evaluaciones con los pacientes del turno correspondiente con cada profesional en las diferentes unidades de cuidados intensivos, se enseñó como debía mantenerse el cierre ocular, como debían aplicarse de manera correcta los colirios y los geles, cuales eran los hallazgos para realizar la interconsulta con el especialista en oftalmología.

\section{Población}

En ambas cohortes, pre y post intervención, fueron incluidos pacientes de 0 a 18 años de edad hospitalizados en las unidades de terapia intensiva pediátrica y cardiovascular, cuyos padres dieron el consentimiento informado en forma verbal Fueron excluidos los pacientes con lesiones oculares conocidas previamente a la hospitalización. 


\section{Definición de las variables}

Fueron estudiadas la edad, sexo, unidad de cuidado intensivo, presencia de apertura ocular anormal, presencia de lesión corneal, tipo de lesión corneal, cumplimiento del organigrama de cuidados oculares.

Definición de las variables:

Apertura ocular anormal: Cierre incompleto del parpado, que permite la exposición del segmento anterior del ojo.

Lesión corneal: alteración en la estructura anatómica de la córnea.

Tipo de lesión corneal: los tipos de lesión pueden ser erosión o ulcera según la profundidad del compromiso corneal y la existencia de infección sobre agregada. La erosión se refiere a un compromiso superficial de las capas de la córnea sin presencia de infección. Ulcera corneal se refiere a un compromiso profundo de la córnea con sobreinfección bacteriana o micótica.

Cumplimiento del organigrama de cuidados oculares: realización o no de los pasos establecidos en el organigrama de cuidados oculares.

\section{Monitoreo de los cuidados oculares y evaluación de las lesiones.}

El monitoreo de los cuidados oculares y la evaluación de las lesiones fueron realizadas por la misma observadora en cada etapa del estudio (dos de las autoras), quiénes previamente fueron capacitadas por una oftalmóloga pediátrica (una de las autoras) del servicio de oftalmología pediátrica del hospital. Todos los días y en diferentes turnos (mañana, tarde y noche) se examinaba el estado ocular de los pacientes (apertura ocular, presencia de lesiones corneales y tipo de lesiones. Se revisaba además las indicaciones de cuidados oculares, la disponibilidad de la medicación (geles, lagrimas oculares).

Los datos fueron consignados en una hoja que contenía las variables

\section{Tamaño de la muestra}

Para conseguir una precisión del 2\% en la estimación de una proporción mediante un intervalo de confianza asintótico normal con corrección para poblaciones finitas al 95\% bilateral, asumiendo que la proporción esperada de lesiones corneales es del $11,8 \%$ y que el tamaño total de la población es de aproximadamente 70, fue necesario incluir 66 participantes como mínimo en ambas etapas del estudio.

Procesamiento de datos y análisis estadístico: Los datos fueron analizados en el sistema SPSSv21, las variables cualitativas son expresados en porcentajes y las continuas con medias con desvío estándar. La asociación de las variables cualitativas se realizó con prueba de chi cuadrado de Pearson o el test exacto de Fisher según necesidad. La asociación de variables cuantitativas se realizó por la T Student los resultados se expresan en riesgo relativo con sus IC al $95 \%$. Se considero un error alfa del $5 \%$.

Asuntos éticos: El estudio fue aprobado por el comité de ética del Hospital.

\section{RESULTADOS}

Durante el periodo de estudio ingresaron 70 pacientes en la cohorte pre - intervención y 120 después de la misma. Los datos demográficos y salas de UTI en el momento del ingreso al estudio puede verse en la Tabla 1.

Tabla 1. Características demográficas, unidad de cuidados intensivos de las cohortes pre y post intervención $\mathrm{N}=190$.

\begin{tabular}{lccc}
\hline & $\begin{array}{c}\text { Pre intervención } \\
\mathbf{n}=\mathbf{7 0}\end{array}$ & $\begin{array}{c}\text { Post intervención } \\
\mathbf{n}=\mathbf{1 2 0}\end{array}$ & $\mathbf{p}$ \\
\hline Edad meses & & & \\
Mediana ( P25 -75) & $3,9 \pm 4,7$ & $31 \pm 47$ & $0,001^{1}$ \\
Sexo & $\mathbf{N}(\mathbf{\%})$ & $\mathbf{N}(\%)$ & \\
$\quad$ Masculino & $51(58,6)$ & $63(52,5)$ & $0,41^{2}$ \\
UCIP* & $48(68,6)$ & $87(72,5)$ & $0,56^{2}$ \\
UCIC** & $22(31,4)$ & $33(27,5)$ & \\
\hline
\end{tabular}

*UCIP: unidad de cuidados intensivos pediatrico **UCIPC: unidad de cuidados intensivos pediátrico cardiovascular 1 TStudent 2 chi cuadrado

La presencia de apertura anormal ocular, así como la presencia de lesiones oculares, así como el cumplimiento del organigrama de cuidados oculares fueron comparados antes y después de la capacitación al personal sanitario. Los resultados se detallan en la tabla 2. 
Tabla 2. Presencia de apertura ocular, lesión ocular y cumplimiento del organigrama en las etapas pre y post intervención. N: 190.

\begin{tabular}{lcccc}
\hline & $\begin{array}{c}\text { Pre intervención } \\
\mathbf{n = 7 0}\end{array}$ & $\begin{array}{c}\text { Post intervención } \\
\mathbf{n = 1 2 0}\end{array}$ & RR (IC 95\%) & p \\
\hline & $\mathrm{N}(\%)$ & $\mathrm{N}(\%)$ & & \\
Apertura ocular anormal & $18(25,7)$ & $19(15,8)$ & $0,77(0,5.0,9)$ & $0,09^{1}$ \\
Lesión corneal & $6(8,6)$ & $3(3,3)$ & $0,3(0,2-0,3)$ & $0,07^{2}$ \\
Cumplimiento del organigrama & $54(77,1)$ & $120(100)$ & $0,3(0,24-0,38)$ & $0,0001^{2}$ \\
\hline
\end{tabular}

1 Chi cuadrado 2 Test Exacto de Fisher

Las lesiones oculares observadas fueron erosión de la córnea en el 8,5\% (6/70) antes de la capacitación y $3,3 \%(3 / 120)$ después de la capacitación $\mathrm{p}=0,17$. Se analizó la asociación de lesión corneal con la apertura de los ojos. En la cohorte previa a la capacitación de las 18 aperturas oculares anormales el 33, 3\% (6/18) presentaron lesiones corneales, mientras que en la cohorte posterior a la capacitación de las 19 aperturas oculares anormales el 15,7\% (3/19) presentaron lesión corneal $\mathrm{p}=0,001$ (Test exacto de Fisher) con un RR de 13 (IC95\% 3,6 -48).

\section{DISCUSIÓN}

La capacitación del personal médico y de enfermería en cuidados oculares disminuyó la frecuencia de lesiones corneales, aunque no fue estadísticamente significativo, significó una disminución del 38\%. Si bien la apertura ocular anormal fue similar en ambos periodos, la presencia de lesiones corneales fue significativamente menor en el periodo post intervención. Esta situación las autoras atribuyen a la mayor disponibilidad e indicación de colirios y geles en las unidades de cuidados intensivos y al cumplimiento del organigrama. Similares resultados se han logrado en la capacitación y monitoreo del cumplimiento de los protocolos de cuidado ocular en los diferentes hospitales, por ejemplo, Carrillo y cols expusieron que el monitoreo del personal que trabaja en las unidades de cuidados intensivos durante el tiempo haría que las lesiones oculares alcancen una frecuencia de $0 \%$, además esta capacitación debe realizarse de manera periódica para lograr que no se presenten lesiones oculares ${ }^{(5)}$. La implementación de programas de educación al personal sanitario sobre los cuidados oculares han tenido un gran impacto en la disminución de las lesiones corneales ${ }^{(8)}$.
La lesión corneal observada en el presente estudio fue menor a la reportada por Hartford J y cols que encontraron $19 \%$ de lesiones corneales por exposición en un grupo de 27 pacientes en la unidad de cuidados intensivos pediátricos. Esta diferencia puede deberse al azar considerando el escaso número de pacientes pediátricos incluidos ${ }^{(9)}$. La lesión corneal en las terapias muchas veces no es reconocida por lo que cada centro hospitalario que maneje pacientes críticos debe desarrollar sus protocolos para el cuidado ocular. Estos protocolos deben ser dinámicos y ser evaluados periódicamente para lograr los objetivos de mantener la córnea en buen estado, evitar infecciones y de esa manera las secuelas visuales del mal manejo ocular ${ }^{(10)}$.

El tipo de lesión ocular más frecuentemente encontrado fue la erosión corneal seguida de la ulcera corneal, resolviéndose en la mayoría de los pacientes en un corto periodo de tiempo aplicando los pasos a seguir en el organigrama, siendo estos hallazgos similares a otros estudios ${ }^{(2,7)}$.

La presencia de apertura ocular anormal es el principal factor de riesgo para la aparición de lesiones corneales y se relaciona con un mayor porcentaje de aparición de lesiones oculares, por lo que debe buscarse el cierre pasivo de los parpados en aquellos pacientes con esta condición y protección con geles y colirios de lágrimas, esto es reportado por varios trabajos publicados ${ }^{(2,47)}$.

Existen otros métodos utilizados en los cuidados oculares de los pacientes críticos como las protecciones con polietileno (cámaras húmedas) o combinaciones de métodos para el cuidado ocular a fin de poder llegar a una prevalencia de lesiones nula $a^{(6,11,12)}$. 
Ahmadinejad y colaboradores comparo en un estudio ramdomizado doble ciego los diferentes métodos de cuidado ocular como cierre ocular, humectación corneal con geles y colirios y las cámaras húmedas con polietileno, en este estudio encontró que la protección aportada por la humectación con geles y colirios es comparable a la cámara húmeda con polietileno ${ }^{(13)}$. Sin embargo Babamohamadi y cols reportó mejor rendimiento con lubricación con vitamina A comparado con las cámaras húmedas ${ }^{(14)}$. Otros estudios reportan a la cámara húmeda como mejor técnica para el cuidado ocular $^{(15,16)}$. En nuestro medio hospitalario se utiliza la lubricación con geles y colirios por el mejor acceso para los pacientes, aunque el costo de estos muchas veces es una limitación este debe estar garantizado en todos los casos.

\section{REFERENCIAS}

1. Skuta G, Cantor L, Weiss J. External Disease and Cornea. 8a ed. San Francisco: McGraw-Hill; 2012.

2. Joyce N. Eye care for intensive care patients: a systematic review. Int J Evid Based Healthc. 2002;6(21):1-5. doi: https://doi.org/10.11124/JBISRIR-2002-388

3. Fernandez L. Prevención de lesiones oculares en el paciente crítico. Valladolid: Universidad de Valladolid; 2016.29 p.

4. Rosenberg JB, Eisen LA. Eye care in the intensive care unit: narrative review and meta-analysis. Crit Care Med. 2008;36(12):3151-3155. doi: https://doi.org/10.1097/CCM.0 b013e31818f0ee7

. Carrillo R, Flores O, Diaz J, Peña C, Maldonado R, Palacios A, et al. Protección ocular en los enfermos internados en la Unidad de Terapia Intensiva. Una propuesta de mejora de calidad y seguridad. Rev. Asoc. Mex. Med. Crít. Ter. Intensiva. 2016;30(1):17-24.

6. Sanchez N, Atescatenco G, Cabrera M. Protección ocular al paciente en estado crítico bajo efectos de sedación. Revista Mexicana de Enfermería Cardiológica. 2011;19(2): 80-82.

7. Alvarenga A, Falci F, Botoni F, Dias J, Machado T. Corneal injuries: incidence and risk factors in the Intensive Care Unit. Rev. Latino-Am. Enfermagem. 2011;19(5):1088-1095.
Este estudio presenta limitaciones como la diferencia de edad de los pacientes en los periodos de pre y post intervención, pero fue la población encontrada en las UCIP. Tampoco se evaluó la severidad de los cuadros de los participantes, el estado de conciencia o la utilización de asistencia respiratoria mecánica. Sin embargo, expuso que la existencia de protocolos de manejo sin capacitación periódica y monitoreo del cumplimiento no es efectiva.

La capacitación constante del personal de las unidades de cuidado intensivo y el seguimiento de los organigramas para el cuidado ocular de los pacientes internados en dichas unidades puede garantizar la reducción de lesiones oculares que posteriormente pueden afectar la visión de los pacientes a largo plazo.

8. Demirel S, Cumurcu T, Firat P, Aydogan MS, Doğanay S. Effective management of exposure keratopathy developed in intensive care units: the impact of an evidence based eye care education programme. Intensive Crit Care Nurs. 2014;30(1):38-44. doi: https://doi.org/ 10.1016/j.iccn.2013.08.001

9. Hartford JB, Bian Y, Mathews PM, De Rojas J, Garg A, Rasool N, et al. Prevalence and Risk Factors of Exposure Keratopathy Across Different Intensive Care Units. Cornea. 2019;38(9):1124-1130. doi: https://doi.org/10.109 7/ICO.0000000000001961

10. Niemi A, Geddie BE, Rajasekaran S, Davis AT, VanDenBosch N, Steenland C, et al. Ocular Surface Disease in a PICU: Incidence and Outcomes With a Dynamic Eye Care Protocol. Pediatr Crit Care Med. 2020;21(4):357-362. doi: https://doi.org/10.1097/PCC.00 00000000002200

11. Carrion M. Revisión crítica: cuidados de enfermería al paciente crítico para reducir incidencias de problemas oculares en UCI. [Tesis]. Chiclayo: Universidad Católica Santo Toribio de Mogrovejo; 2015.

12. Bates J, Dwyer R, O'Toole L, Kevin L, O'Hegarty N, Logan P. Corneal protection in critically ill patients: a randomized controlled trial of three methods. Clin Intensive Care. 2004;15(1):23-26. doi: https://doi.org/10 $.3109 / 09563070410001661759$ 
13. Ahmadinejad M, Karbasi E, Jahani Y, Ahmadipour M, Soltaninejad M, Karzari Z. Efficacy of Simple Eye Ointment, Polyethylene Cover, and Eyelid Taping in Prevention of Ocular Surface Disorders in Critically Ill Patients: A Randomized Clinical Trial. Crit Care Res Pract. 2020; 2020:6267432. doi: https://doi.org/10.1155/2020 /6267432

14. Babamohamadi H, Nobahar M, Razi J, Ghorbani R. Comparing Vitamin A and Moist Chamber in Preventing Ocular Surface Disorders. Clin Nurs Res. 2018;27(6):714729. doi: https://doi.org/10.1177/1054773817695618
15 Kalhori RP, Ehsani S, Daneshgar F, Ashtarian H, Rezaei M. Different Nursing Care Methods for Prevention of Keratopathy Among Intensive Care Unit Patients. Glob J Health Sci. 2015;8(7):212-7. doi: https://doi.org/10.5539 /gjhs.v8n7p212

16. Grixti A, Sadri M, Edgar J, Datta AV. Common ocular surface disorders in patients in intensive care units. Ocul Surf. 2012;10(1):26-42. doi: https://doi.org/10.1016/j.jtos.2011.10.001 